

\title{
Spherical angular spectrum and the fractional order Fourier transform
}

\author{
Pierre Pellat-Finet, Pierre-Emmanuel Durand, Eric Fogret
}

\section{To cite this version:}

Pierre Pellat-Finet, Pierre-Emmanuel Durand, Eric Fogret. Spherical angular spectrum and the fractional order Fourier transform. Optics Letters, 2006, 31 (23), pp.3429-3431. hal-00907972

\section{HAL Id: hal-00907972 \\ https://hal.science/hal-00907972}

Submitted on 22 Nov 2013

HAL is a multi-disciplinary open access archive for the deposit and dissemination of scientific research documents, whether they are published or not. The documents may come from teaching and research institutions in France or abroad, or from public or private research centers.
L'archive ouverte pluridisciplinaire HAL, est destinée au dépôt et à la diffusion de documents scientifiques de niveau recherche, publiés ou non, émanant des établissements d'enseignement et de recherche français ou étrangers, des laboratoires publics ou privés. 


\title{
Spherical angular spectrum and the fractional order Fourier transform
}

\author{
Pierre Pellat-Finet, Pierre-Emmanuel Durand, and Éric Fogret \\ Groupe d'Optique Théorique et Appliquée, Université de Bretagne Sud, B.P. 92116, 56321 Lorient Cedex, France \\ Received June 12, 2006; revised September 4, 2006; accepted September 13, 2006; \\ posted September 15, 2006 (Doc. ID 71928); published November 9, 2006 \\ The notion of a spherical angular spectrum leads to the decomposition of the field amplitude on a spherical \\ emitter into a sum of spherical waves that converge onto the Fourier sphere of the emitter. Unlike the usual \\ angular spectrum, the spherical angular spectrum is propagated as the field amplitude, in a way that can be \\ expressed by a fractional order Fourier transform. (C) 2006 Optical Society of America \\ OCIS codes: $070.2580,070.2590$.
}

According to a scalar theory of diffraction, the field amplitude transfer from a plane emitter $\mathcal{P}$ to a plane receiver $\mathcal{Q}$ at a distance $D$ can be written as the convolution product ${ }^{1}$

$$
U_{Q}(\mathbf{s})=\int_{\mathbb{R}^{2}} h(\mathbf{s}-\mathbf{r}) U_{P}(\mathbf{r}) d \mathbf{r},
$$

where $\lambda$ is the wavelength, $U_{P}(\mathbf{r})$ the field amplitude at the point $\mathbf{r}$ on $\mathcal{P}, U_{Q}(\mathbf{s})$ the field amplitude on $\mathcal{Q}$, and $h$ the spatial impulse function associated with the field propagation. Apart from a constant phase factor, and in the limit of a second-order approximation, the expression for $h$ is

$$
h(\mathbf{r})=(i / \lambda D) \exp \left[-(i \pi / \lambda D) r^{2}\right],
$$

where $r^{2}=\|\mathbf{r}\|^{2}$.

The same transfer can be expressed as a simple product in the Fourier space if we introduce the angular spectra ${ }^{1} A_{P}$ of the field on $\mathcal{P}$ and $A_{Q}$ on $\mathcal{Q}$. If $\mathcal{F}_{\pi / 2}\left[U_{P}\right]$ denotes the Fourier transform of $U_{P}$, we define the angular spectrum on $\mathcal{P}$ by

$$
\begin{aligned}
A_{P}(\boldsymbol{\Phi}) & =\frac{1}{\lambda^{2}} \mathcal{F}_{\pi / 2}\left[U_{P}\right]\left(\frac{\boldsymbol{\Phi}}{\lambda}\right) \\
& =\frac{1}{\lambda^{2}} \int_{\mathbb{R}^{2}} \exp \left(\frac{2 i \pi}{\lambda} \boldsymbol{\Phi} \cdot \mathbf{r}\right) U_{P}(\mathbf{r}) \mathrm{d} \mathbf{r},
\end{aligned}
$$

where $\boldsymbol{\Phi}=\lambda \mathbf{F}$ (F is a spatial frequency and $\boldsymbol{\Phi}$ an angular spatial frequency). We define $\Phi=\|\Phi\|$, and we use

$$
H(\boldsymbol{\Phi})=\mathcal{F}_{\pi / 2}[h](\boldsymbol{\Phi} / \lambda)=\exp \left[(i \pi D / \lambda) \Phi^{2}\right],
$$

so that Eq. (1) leads us to

$$
A_{Q}(\boldsymbol{\Phi})=H(\boldsymbol{\Phi}) A_{P}(\boldsymbol{\Phi}) .
$$

The former descriptions of the field transfer are summed up in the following diagram:

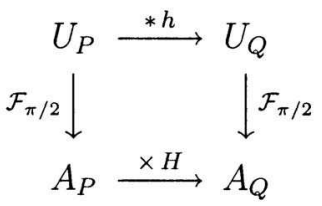

Diagram (6) is not commutative: to pass from $U_{P}$ to $A_{Q}$ we perform a Fourier transform followed by a multiplication, or a convolution product followed by a Fourier transform; the operation order cannot be inverted.

We shall introduce the notion of spherical angular spectrum and explain how diagram (6) becomes one that is commutative. We refer to spherical emitters and receivers: they are portions of spheres with axial symmetry. A spherical surface $\mathcal{A}$ has a vertex $\Omega$ and a center of curvature $C$ : its radius of curvature is $R_{A}$ $=\Omega C$ and can be positive or negative according to the surface convexity. A point $M$ on $\mathcal{A}$ is represented by the vector $\mathbf{r}=\Omega m$, where $m$ is the orthogonal projection of $M$ onto the plane tangent to $\mathcal{A}$ at $\Omega$. $U_{A}(\mathbf{r})$ denotes the field amplitude at $M$ on $\mathcal{A}$, and we define the spherical angular spectrum on $\mathcal{A}$ as

$$
S_{A}(\boldsymbol{\Phi})=\left(1 / \lambda^{2}\right) \mathcal{F}_{\pi / 2}\left[U_{A}\right](\boldsymbol{\Phi} / \lambda) .
$$

We invert Eq. (7) and obtain

$$
U_{A}(\mathbf{r})=\int_{\mathbb{R}^{2}} \exp \left(-\frac{2 i \pi}{\lambda} \boldsymbol{\Phi} \cdot \mathbf{r}\right) S_{A}(\boldsymbol{\Phi}) \mathrm{d} \boldsymbol{\Phi},
$$

so that the field amplitude on $\mathcal{A}$ is the sum of elementary field amplitudes $\exp (-2 i \pi \boldsymbol{\Phi} \cdot \mathbf{r} / \lambda)$, weighted by the spherical angular spectrum. To interpret such a field amplitude, we introduce the Fourier sphere $\mathcal{F}$ of $\mathcal{A}$ : its vertex is $C$, and its center of curvature is $\Omega$ (Fig. 1). Its radius of curvature is $R_{F}=C \Omega=-R_{A}$.

We know ${ }^{2}$ that the field amplitudes on $\mathcal{A}$ and $\mathcal{F}$ are related by a Fourier transform [as Eq. (10) will

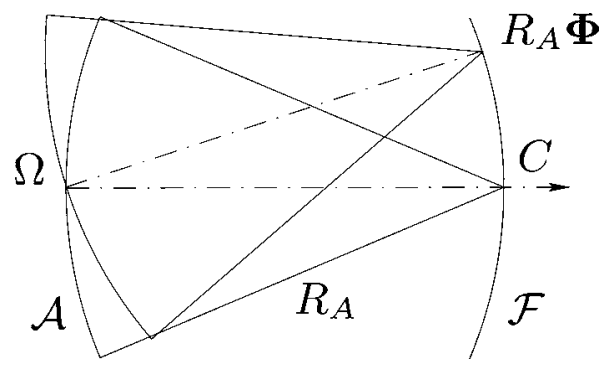

Fig. 1. Spherical wave converging at the point $R_{A} \boldsymbol{\Phi}$ on the Fourier sphere $\mathcal{F}$ generates a field amplitude on $\mathcal{A}$ equal to $\exp (-2 i \pi \boldsymbol{\Phi} \cdot \mathbf{r} / \lambda)$. 
show]. If $\delta$ denotes the Dirac distribution, a luminous point located at $R_{A} \Phi$ on $\mathcal{F}$ is represented by $\delta\left(\mathbf{s}-R_{A} \boldsymbol{\Phi}\right)$, up to a dimensional factor, and generates a field amplitude on $\mathcal{A}$ proportional to $\exp (-2 i \pi \Phi \cdot \mathbf{r} / \lambda)$. We use the reciprocity theorem ${ }^{3}$ and conclude that $\exp (-2 i \pi \Phi \cdot \mathbf{r} / \lambda)$ represents the amplitude of the field generated on $\mathcal{A}$ by the spherical wave converging at the point $R_{A} \Phi$ on $\mathcal{F}$. The wave can also be seen as a tilted spherical wave as indicated in Fig. 1.

We examine now how the spherical angular spectrum is propagated. The field amplitude transfer from the spherical emitter $\mathcal{A}$ to the spherical receiver $\mathcal{B}$ at distance $D$ can be written as ${ }^{4}$

$$
\begin{aligned}
U_{B}(\mathbf{s})= & \frac{i}{\lambda D} \exp \left[-\frac{i \pi s^{2}}{\lambda}\left(\frac{1}{D}+\frac{1}{R_{B}}\right)\right] \\
& \times \int_{\mathbb{R}^{2}} \exp \left(\frac{2 i \pi}{\lambda D} \mathbf{s} \cdot \mathbf{r}\right) \\
& \times \exp \left[\frac{i \pi r^{2}}{\lambda}\left(\frac{1}{R_{A}}-\frac{1}{D}\right)\right] U_{A}(\mathbf{r}) \mathrm{d} \mathbf{r} .
\end{aligned}
$$

If $\mathcal{B}=\mathcal{F}$ (the Fourier sphere of $\mathcal{A}$ ), Eq. (9) reduces to

$$
U_{F}(\mathbf{s})=\left(i / \lambda R_{A}\right) \mathcal{F}_{\pi / 2}\left[U_{A}\right]\left(\mathbf{s} / \lambda R_{A}\right),
$$

and we obtain

$$
S_{F}(\boldsymbol{\Phi})=\frac{1}{\lambda^{2}} \mathcal{F}_{\pi / 2}\left[U_{F}\right]\left(\frac{\boldsymbol{\Phi}}{\lambda}\right)=\frac{i R_{A}}{\lambda} \mathcal{F}_{\pi / 2}\left[S_{A}\right]\left(\frac{R_{A} \boldsymbol{\Phi}}{\lambda}\right) .
$$

The transfer of the spherical angular spectrum from an emitter to its Fourier sphere is expressed by a Fourier transform, as well as the field amplitude.

If $\mathcal{A}$ and $\mathcal{B}$ are concentric spheres, then $D=R_{A}$ $-R_{B}$. We introduce $\kappa=R_{B} / R_{A}$ and the function $h_{B A}$ such that

$$
h_{B A}(\mathbf{r})=\frac{i}{\lambda D} \exp \left[(-i \pi \kappa / \lambda D) r^{2}\right] .
$$

Equation (9) then becomes

$$
U_{B}(\mathbf{s})=\int_{\mathbb{R}^{2}} h_{B A}\left(\frac{\mathbf{s}}{\kappa}-\mathbf{r}\right) U_{A}(\mathbf{r}) \mathrm{d} \mathbf{r}=\left[h_{B A} * U_{A}\right]\left(\begin{array}{l}
\mathbf{s} \\
\kappa
\end{array}\right)
$$

and involves a convolution product. We conclude that the spherical angular spectra on $\mathcal{A}$ and $\mathcal{B}$ are such that

$$
S_{B}(\boldsymbol{\Phi})=\kappa \exp \left[(i \pi \kappa D / \lambda) \Phi^{2}\right] S_{A}(\kappa \boldsymbol{\Phi}) .
$$

The propagation between two concentric spheres acts as a linear filter.

For arbitrary emitter $\mathcal{A}$ and receiver $\mathcal{B}$ we introduce the sphere $\mathcal{A}^{\prime}$ concentric to $\mathcal{A}$ that passes by the center of $\mathcal{B}$, and the sphere $\mathcal{B}^{\prime}$ concentric to $\mathcal{B}$ that passes by the center of $\mathcal{A}$ (Fig. 2). Clearly $\mathcal{B}^{\prime}$ is the Fourier sphere of $\mathcal{A}^{\prime}$, so that the field transfer from $\mathcal{A}$ to $\mathcal{B}$ can be decomposed into a linear filtering from $\mathcal{A}$ to $\mathcal{A}^{\prime}$ followed by a Fourier transform from $\mathcal{A}^{\prime}$ to $\mathcal{B}^{\prime}$ and a linear filtering from $\mathcal{B}^{\prime}$ to $\mathcal{B}$. The radius of $\mathcal{A}^{\prime}$ is

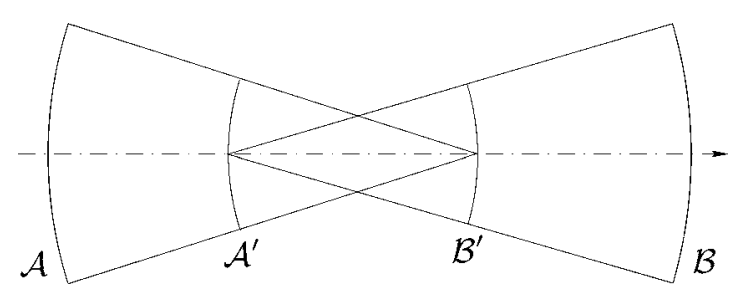

Fig. 2. Field transfer from $\mathcal{A}$ to $\mathcal{B}$ can be decomposed into a linear filtering from $\mathcal{A}$ to $\mathcal{A}^{\prime}$ followed by a Fourier transform from $\mathcal{A}^{\prime}$ to $\mathcal{B}^{\prime}$ and another linear filtering from $\mathcal{B}^{\prime}$ to $\mathcal{B}$.

opposite of the radius of $\mathcal{B}^{\prime}: R_{A^{\prime}}=R_{A}-D-R_{B}=-R_{B^{\prime}}$. We use $\kappa^{\prime}=R_{A^{\prime}} / R_{A}, \kappa^{\prime \prime}=R_{B} / R_{B^{\prime}}, D^{\prime}=D+R_{B}$, and $D^{\prime \prime}$ $=D-R_{A}$, and we deduce from Eqs. (11) and (14) that

$$
\begin{aligned}
S_{B}(\boldsymbol{\Phi})= & \frac{i R_{A^{\prime}} \kappa^{\prime \prime}}{\lambda \kappa^{\prime}} \exp \left(\frac{i \pi \kappa^{\prime \prime} D^{\prime \prime} \Phi^{2}}{\lambda}\right) \int_{\mathbb{R}^{2}} \exp \left(\frac{i \pi D^{\prime} \Phi^{\prime 2}}{\lambda \kappa^{\prime}}\right) \\
& \times \exp \left(\frac{2 i \pi \kappa^{\prime \prime} R_{A^{\prime}}}{\lambda \kappa^{\prime}} \boldsymbol{\Phi} \cdot \boldsymbol{\Phi}^{\prime}\right) S_{A}\left(\boldsymbol{\Phi}^{\prime}\right) \mathrm{d} \boldsymbol{\Phi}^{\prime} .
\end{aligned}
$$

Equation (15) has the same form as Eq. (9): quadratic phase factors in front of and inside the integral, Fourier kernel. Unlike the usual angular spectrum, the spherical angular spectrum propagates as the field amplitude.

Since we know ${ }^{2,4}$ that the field transfer from a spherical emitter $\mathcal{A}$ to a spherical receiver $\mathcal{B}$ can be represented by a fractional order Fourier transform $\mathcal{F}_{\alpha}$ [Eq. (16) provides a definition], the similarity between Eqs. (15) and (9) leads us to express the transfer of the spherical angular spectrum by using a fractional Fourier transform. For that purpose, we define the fractional Fourier transform of order $\alpha$ (a complex number) of the two-dimensional function $f$ by $^{5}$

$$
\begin{aligned}
\mathcal{F}_{\alpha}[f]\left(\boldsymbol{\rho}^{\prime}\right)= & \frac{i e^{-i \alpha}}{\sin \alpha} \exp \left(-i \pi \rho^{\prime 2} \cot \alpha\right) \\
& \times \int_{\mathbb{R}^{2}} \exp \left(-i \pi \rho^{2} \cot \alpha\right) \\
& \times \exp \left(\frac{2 i \pi \boldsymbol{\rho}^{\prime} \cdot \boldsymbol{\rho}}{\sin \alpha}\right) f(\boldsymbol{\rho}) \mathrm{d} \boldsymbol{\rho} .
\end{aligned}
$$

The standard Fourier transform is obtained by setting $\alpha=\pi / 2$ in Eq. (16).

Equation (9) can be expressed as a fractional Fourier transform of order $\alpha$ as follows. We choose $\alpha(-\pi<\alpha<\pi)$ and $\epsilon$ (an auxiliary parameter) such that

$$
\begin{array}{cl}
\cot ^{2} \alpha=\frac{\left(D+R_{B}\right)\left(R_{A}-D\right)}{D\left(D-R_{A}+R_{B}\right)}, & \alpha D \geqslant 0, \\
\epsilon^{2}=\frac{D\left(D+R_{B}\right)}{\left(R_{A}-D\right)\left(D-R_{A}+R_{B}\right)}, & \epsilon R_{A}>0 .
\end{array}
$$

(We assume that $\alpha$ and $\epsilon$ are real numbers. We refer to a recent publication ${ }^{6}$ for handling complex param- 
eters.) We define scaled variables and scaled field amplitudes on $\mathcal{A}$ and on $\mathcal{B}$ by

$$
\begin{gathered}
\boldsymbol{\rho}=\frac{\mathbf{r}}{\left(\lambda \epsilon R_{A}\right)^{1 / 2}}, \quad V_{A}(\boldsymbol{\rho})=U_{A}\left[\left(\lambda \epsilon R_{A}\right)^{1 / 2} \boldsymbol{\rho}\right], \\
\boldsymbol{\sigma}=\frac{(\cos \alpha+\epsilon \sin \alpha) \mathbf{s}}{\left(\lambda \epsilon R_{A}\right)^{1 / 2}}, \\
V_{B}(\boldsymbol{\sigma})=U_{B}\left[\frac{\left(\lambda \epsilon R_{A}\right)^{1 / 2} \boldsymbol{\sigma}}{\cos \alpha+\epsilon \sin \alpha}\right] .
\end{gathered}
$$

Equation (9) becomes

$$
V_{B}(\boldsymbol{\sigma})=e^{i \alpha}(\cos \alpha+\epsilon \sin \alpha) \mathcal{F}_{\alpha}\left[V_{A}\right](\boldsymbol{\sigma})
$$

and shows how the scaled field amplitude on $\mathcal{B}$ is related to the fractional Fourier transform of order $\alpha$ of the scaled field amplitude on $\mathcal{A}$.

Since we are looking for a commutative diagram, and since the composition law of fractional Fourier transforms demands that $\mathcal{F}_{\alpha} \circ \mathcal{F}_{\pi / 2}=\mathcal{F}_{\pi / 2} \circ \mathcal{F}_{\alpha}$, we expect the transfer of the spherical angular spectrum from $\mathcal{A}$ to $\mathcal{B}$ to be expressed by a fractional Fourier transform whose order should be equal to $\alpha$. We prove that this holds true indeed. Since $\alpha$ has been defined with respect to the field amplitude transfer, we only have to define scaled variables and scaled angular spectra $\Sigma_{A}$ and $\Sigma_{B}$. According to Eq. (15), the angular spatial frequencies are $\boldsymbol{\Phi}^{\prime}$ on $\mathcal{A}$ and $\boldsymbol{\Phi}$ on $\mathcal{B}$, and we choose

$$
\begin{gathered}
\boldsymbol{\phi}^{\prime}=\left(\frac{\epsilon R_{A}}{\lambda}\right)^{1 / 2} \boldsymbol{\Phi}^{\prime}, \quad \boldsymbol{\phi}=\left(\frac{\epsilon R_{A}}{\lambda}\right)^{1 / 2} \frac{\boldsymbol{\Phi}}{\cos \alpha+\epsilon \sin \alpha}, \\
\Sigma_{A}\left(\boldsymbol{\phi}^{\prime}\right)=S_{A}\left[\left(\lambda / \epsilon R_{A}\right)^{1 / 2} \boldsymbol{\phi}^{\prime}\right], \\
\Sigma_{B}(\boldsymbol{\phi})=S_{B}\left[\left(\lambda / \epsilon R_{A}\right)^{1 / 2}(\cos \alpha+\epsilon \sin \alpha) \boldsymbol{\phi}\right] .
\end{gathered}
$$

Thus Eq. (15) can be written as

$$
\Sigma_{B}(\boldsymbol{\phi})=\left[e^{i \alpha} /(\cos \alpha+\epsilon \sin \alpha)\right] \mathcal{F}_{\alpha}\left[\Sigma_{A}\right](\boldsymbol{\phi}) .
$$

The transfer of the spherical angular spectrum can be expressed by a fractional order Fourier transform, as well as the field amplitude transfer, with the same order.

From the definitions of scaled field amplitudes $V_{A}$ and $V_{B}$ and scaled angular spectra $\Sigma_{A}$ and $\Sigma_{B}$, we deduce

$$
\begin{gathered}
\Sigma_{A}\left(\boldsymbol{\phi}^{\prime}\right)=\left(\epsilon R_{A} / \lambda\right) \hat{V}_{A}\left(\boldsymbol{\phi}^{\prime}\right), \\
\Sigma_{B}(\boldsymbol{\phi})=\left\{\epsilon R_{A} /\left[\lambda(\cos \alpha+\epsilon \sin \alpha)^{2}\right]\right\} \hat{V}_{B}(\boldsymbol{\phi}),
\end{gathered}
$$

where $\hat{V}_{A}$ and $\hat{V}_{B}$ are the Fourier transforms of $V_{A}$ and $V_{B}$. Equation (25) leads to

$$
\hat{V}_{B}(\boldsymbol{\phi})=e^{i \alpha}(\cos \alpha+\epsilon \sin \alpha) \mathcal{F}_{\alpha}\left[\hat{V}_{A}\right](\boldsymbol{\phi}),
$$

which corresponds to Eq. (21).

We come to the following commutative diagram, which is applied to scaled field amplitudes and their Fourier transforms [a factor $e^{i \alpha}(\cos \alpha+\epsilon \sin \alpha)$ has been omitted]:

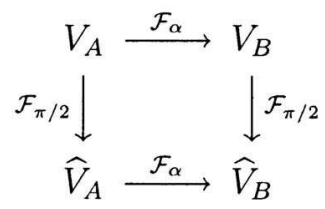

Obviously diagram (6) cannot be reduced to diagram (29). We conclude that the notion of angular spectrum, as it is usually defined, ${ }^{1}$ does not fit in with fractional Fourier optics.

In conclusion, we remark that the notion of spherical angular spectrum can be used to solve propagation problems in the framework of a scalar theory of diffraction: for example, it can be applied to image formation. ${ }^{7}$ It offers a substitute for the usual angular spectrum and affords the application of the fractional order Fourier transform in solving these problems.

P. Pellat-Finet (pierre-pellat-finet@univ-ubs.fr) and É. Fogret are also with the Département d'Optique, UMR CNRS 6082, École Nationale Supérieure des Télécommunications de Bretagne, Brest, France.

\section{References}

1. J. W. Goodman, Introduction to Fourier Optics (McGraw-Hill, 1996).

2. P. Pellat-Finet and G. Bonnet, Opt. Commun. 111, 141 (1994).

3. M. Born and E. Wolf, Principles of Optics (Cambridge U. Press, 1999).

4. P. Pellat-Finet, Opt. Lett. 19, 1388 (1994).

5. V. Namias, J. Inst. Math. Appl. 25, 241 (1980).

6. P. Pellat-Finet and E. Fogret, Opt. Commun. 258, 103 (2006).

7. P. Pellat-Finet and P. E. Durand, C. R. Phys. 7, 457 (2006). 